\title{
TEAM SITUATION AWARENESS USING WEB-BASED FUZZY GROUP DECISION SUPPORT SYSTEMS
}

\author{
Jie LU, Guangquan ZHANG, Fengjie WU \\ Faculty of Information Technology, University of Technology Sydney \\ PO Box 123, Broadway, NSW 2007, Australia \\ \{jielu, zhangg, fengjiew\}@it.uts.edu.au \\ Received October $28^{\text {th }}, 2007$ \\ Revised November $12^{\text {th }}, 2007$
}

\begin{abstract}
Situation awareness (SA) is an important element to support responses and decision making to crisis problems. Decision making for a complex situation often needs a team to work cooperatively to get consensus awareness for the situation. Team SA is characterized including information sharing, opinion integration and consensus SA generation. In the meantime, various uncertainties are involved in team SA during information collection and awareness generation. Also, the collaboration between team members may be across distances and need web-based technology to facilitate. This paper presents a web-based fuzzy group decision support system (WFGDSS) and demonstrates how this system can provide a means of support for generating team SA in a distributed team work context with the ability of handling uncertain information.
\end{abstract}

Keywords: Situation awareness, uncertain information processing, group decision-making, fuzzy sets, web-based decision support systems.

\section{Introduction}

Critical situation management not only focuses on the immediate aspect of a disaster, but also pays more attention to finding ways to avoid crisis problems in the first place and preparing suitable responses to minimize the loss for those that undoubtedly will occur, such as fires, floods, epidemic, and even terrorism. This mission requires technical support in effectively analyzing information of a situation, providing assessment results for emergency management officers and supporting their awareness and suitable decisions. Situation awareness (SA) is considered as an important element in completing this mission. SA is defined by Endsley ${ }^{1}$ as "the perception of elements in the environment, the comprehension of their meaning in terms of task goals, and the projection of their status in the near future". The process of achieving SA is called situation analysis or situation assessment, which is based on acquired situation information that may be uncertain. Awareness information for a situation is derived as the results of situation assessment.

Since SA is regarded as a dynamic and collaborative process, and assessing a situation is often required in a team, ${ }^{2,3}$ reaching an overall SA across a team is a pre-emptive requirement for team SA. Typically, collaboration, information sharing and SA aggregation/integration are the main components of team SA generation process. Normally, in a team, each member holds its own component(s) of SA, which may be very different from other member's view of the situation, and should be aware of the overall situation, other team members, and their actions involved ${ }^{4}$. When team members collaborate in a face-to-face environment, they can easily share 
information obtained for a situation from others. Although each individual may have personal prior knowledge, experience, and opinions, the shared physical environment provides a common reference to support the communication among team members and to develop an information sharing and integration working environment. When team members collaborate across distances, each individual's $\mathrm{SA}$, including the awareness of the local and remote situations, would have to be facilitated and supported by suitable information technologies ${ }^{5,6}$. The web-based group decision support systems (GDSS) provide a means to help a team achieve consensus awareness, since such systems have capability in online information sharing, interaction, and integration. However, information and awareness acquired is often inaccuracy. Furthermore, team members often have conflict preferences, and their data is often described by linguistic terms, in assessing and sensing a situation. Therefore, uncertain information representation and aggregation functions are required in the web-based GDSS for supporting a team's SA generation.

This study considers the challenge of building a web-based fuzzy GDSS that can aggregate uncertain information and generate SA for a distributed team by collaborating individuals' situation assessment and awareness over the web. The rest of this paper is organized as follows. Section 2 discusses the requirements of team SA and provides motivation for the use of both web-based GDSS and uncertain information process techniques. Section 3 describes a fuzzy-sets based multi-criteria group decision aggregation method and a web-based fuzzy GDSS, called WFGDSS. Section 4 demonstrates how the WFGDSS can support team SA generation illustrated by a case based example. Finally, Section 5 concludes the report and outlines the directions of our further work.

\section{Requirements of Team Situation Awareness}

Situation awareness has been considered as a phenomenon that refers to extract environmental information, and then integrate it with previous knowledge to direct further perception and anticipate fu- ture events ${ }^{1,7}$. It is an understanding of the state of the environment and provides the primary basis for subsequent decision making and performance in the operation of complex and dynamic systems. ${ }^{8}$ Team SA is commonly used in the human-computer interaction community where the concerns are to design computer interfaces so that a human operator can achieve SA in a timely fashion. It is also used in the data fusion community where it is more commonly referred to as situation assessment. ${ }^{9}$ SA has been largely studied as an important element in diverse military and pilot systems using observation, experiments, and empirical methods. It is recently developed in critical decision making and early warning systems. To develop more effective applications of SA in these fields, three main issues below that influence situation assessment and awareness creation are to be solved.

(i) Situation information uncertainty: Team members collect data, generate awareness for a situation, and share them with other members aiming to get consensus awareness for the situation in the team. Two basic functions are required to support the generation of team SA. The first is the representation of the data collected about a situation. In a real world people often only imprecisely or ambiguously know a situation and use uncertain information to present it, for examples, 'very high', 'high' and 'very low'. The second is the approaches or tools for assessing the situation. As team SA has to be generated through aggregating these imprecise and inaccuracy information and opinions, fuzzy information processing techniques, particularly fuzzy sets based linguistic term process approaches, are suitable.

(ii) SA inconsistency within team members: Assessing or controlling complex and dynamic situations, such as an emergency co-ordination among several large organizations, is beyond the competence of one single individual. Instead a team works cooperatively to coordinate and control the environment. Since each team member possesses his/her own SA, the level of overall SA across the team becomes an important issue, possibly leading to performance errors in team SA. An example can be found in the context of a building security control room. Several security officers need to know cer- 
tain pieces of information to safely and effectively complete a work process. If one person acting as a supervisor is aware of the critical information, but another person in direct control of the process is not, the SA of the team may be deficient. Consequently, performance and system safety may suffer from this case. This is also a typical case of SA inconsistency. Team members communicate each other to describe their identifications and judgments for a situation in attempting to reach a consensus SA. In a sequence processing, relevant SA information is passed on to the next person that may produce fewer uncertain hypotheses. Parallel processing would make team members develop different situation models that at the end might lead team members to talk about different conceptions of the ambiguous situation. Therefore, a suitable team situation assessment collaboration platform and an aggregation method are necessary in order to get an overall SA across a team.

(iii) Distributed environment: In collaborative team work, perceiving, recognizing, and understanding activities of other members through sharing SA are basic requirements. Several possibilities exist for sharing SA: each team member might have the same SA, which could be accurate or inaccurate, or each team member might have different SA, in which case either one is accurate and one is not, or they are both inaccurate. Communication is the key to team SA by reducing the occurrence of discrepancies in shared SA. Shared SA is as the intersection of elements within an environment upon which multiple members must develop SA for accomplishing individual sub-goals leading to achievement of the overall team goal. It should be noted that the accuracy of team SA depends upon the accuracy of each team member's SA; if any team member has poor SA, then the team SA may become poor. ${ }^{10}$ In addition, complete SA, in which a member has access to all possible awareness about another member, can better support decision than partial SA, and it often combines with shared SA in a distributed environment. ${ }^{11}$

The three issues identified on above propose crucial requirements for web-based GDSS which have uncertain information representation and aggrega- tion capability for team SA generation in a distributed environment.

\section{A Web-based Fuzzy Group Decision Support System}

\subsection{Group decision support systems and fuzzy sets}

A GDSS is characterized by being adapted for a group of people who collaborate using a common computer network such as the web to support integrated systems thinking for complex decision making. ${ }^{12,13}$ Various GDSSs have been used as communication support systems in electronic meetings, project evaluations and recently emergency situations. ${ }^{3}$ The communication process in emergencies is usually implemented by two types of communication support methods; one is a communication support system based on the communication model which considers human related factors such as "competence", "duty", "responsibility" and "knowledge", and the other is coordinated interfaces to visualize the gap between intention and situation. ${ }^{14}$ This study mainly focuses on the first type.

Fuzzy set techniques proposed by Zadeh ${ }^{15}$ have been well applied in the area of multi-criteria group decision-making to deal with uncertain issues, particularly linguistic terms, ${ }^{16}$ involved in generating a consensus opinion (solution) for a decision group. ${ }^{17,18,19}$ Our previous study ${ }^{20,21}$ identified three factors that influence the assessment of utility of alternatives and the deriving of a group consensus opinion and decision:

(1) individuals' roles (weights) in the ranking of the satisfactory decisions;

(2) individuals' preferences for alternatives; and

(3) individuals' judgments on criteria for assessing these alternatives.

We also proposed a framework to represent group members' perspectives in order to minimize their confliction in a decision-making process. ${ }^{13}$ Based on these research results, a fuzzy multi-criteria group 
decision aggregation method was proposed, which uses general fuzzy numbers to present three sets of linguistic terms described in above identified three uncertain factors. A consensus group decision (or called a satisfactory solution) which is in the most acceptable degree of the group can be obtained by applying the method.

\subsection{A fuzzy multi-criteria group decision aggregation method}

This method consists of ten steps within three stages.

Let $P=\left\{P_{1}, P_{2}, \ldots, P_{n}\right\}, n \geqslant 2$, be a given finite set of decision makers to select a satisfactory solution from alternatives.

Stage 1: Alternatives, criteria, and individual weights determination

Step 1: When a decision problem is proposed in a group, each member can raise possible alternatives. Let $S^{\#}=\left\{S_{1}^{p_{1}}, S_{2}^{p_{1}}, \ldots, S_{m}^{p_{1}}, \ldots, S_{1}^{p_{n}}, S_{2}^{p_{n}}, \ldots, S_{m}^{p_{n}}\right\}$, where $S_{j}^{p_{i}}$ is the $j^{\text {th }}$ alternative for the decision problem raised by group member $P_{i}$. Through communication, $S=\left\{S_{1}, S_{2}, \ldots, S_{m}\right\}, m \geqslant 2$ is selected from $S^{\#}$ as alternatives for the decision problem in this group.

Step 2: Each group member $P_{k}(k=1,2, \ldots, n)$ can propose $a_{k}$ criteria $\left(C_{1}^{k}, C_{2}^{k}, \ldots, C_{a_{k}}^{k}\right)$ for assessing these alternatives. All members' criteria are put into a criterion pool and top-t criteria, $C=$ $\left\{C_{1}, C_{2}, \ldots, C_{t}\right\}$, are chosen as criteria for the decision problem in the group. Chosen through discussions and compromise among group members, these top-t criteria will reflect the understanding and preference of the whole group.

Step 3: As group members may have different degrees of influence for the selection of the group satisfactory solution, the relative importance of each decision maker may not equal in a decision group. Therefore, each member is assigned with a weight that is described by a linguistic term $\widetilde{v}^{k}$, $k=1,2, \ldots, n$. These terms are determined through discussions in the group or assigned by a higher management level (say, the group leader) at the beginning of the decision process. Possible linguistic terms used are shown in Table 1.

Stage 2: Individual preference generation
Step 4: Each decision maker $P_{k}(k=1,2, \ldots, n)$ is required to express their preference for criteria by a pairwise comparison of the relative importance of these criteria of AHP method. ${ }^{22}$

Table 1. Linguistic terms for describing weights of decision makers

\begin{tabular}{|l|c|}
\hline Linguistic terms & Fuzzy numbers \\
\hline Normal & $c_{1}$ \\
\hline Important & $c_{2}$ \\
\hline More important & $c_{3}$ \\
\hline Most important & $c_{4}$ \\
\hline
\end{tabular}

An initial pairwise comparison matrix $E=$ $\left[\tilde{e}_{i j}^{k}\right]_{t \times t}$ is firstly established, where $\widetilde{e}_{i j}^{k}$ represents the quantified judgments on pairs of criteria $C_{i}$ and $C_{j}$ $(i, j=1,2, \ldots, t, i \neq j)$. The comparison scale belongs to a set of linguistic terms that contain various degrees of preferences required by decision makers $P_{k}(k=1,2, \ldots, n)$, or take a value ' $*$ '. The linguistic terms are shown in Table 2. Character ' $*$ ' represents that decision maker $P_{k}(k=1,2, \ldots, n)$ does not know or cannot compare the relative importance of criteria $C_{i}$ and $C_{j}$.

Consistent weights $w_{i}^{k}(i=1,2, \ldots, t)$ for every criterion can be determined by calculating the geometric mean of each row of the matrix $\left[\widetilde{e}_{i j}^{k}\right]_{t \times t}$ where $e_{i j}^{k}\left(j=1,2, \ldots, i_{k}\right)$ is not ' $*$ ', and then the resulting fuzzy numbers are normalized and denoted as $\widetilde{w}_{1}^{k}$, $\widetilde{w}_{2}^{k}, \ldots, \widetilde{w}_{i}^{k}$, where $\widetilde{w}_{i}^{k} \in F_{T}^{*}(R)$ and

$$
\widetilde{w}_{i}^{k}=\frac{w_{i}^{k}}{\sum_{i=1}^{t} w_{i 0}^{k}} \text { for } i=1,2, \ldots, t ; k=1,2, \ldots, n
$$

Step 5: Against every selection criterion $C_{j}(j=$ $1,2, \ldots, t)$, a belief level can be introduced to express the possibility of selecting a solution Si under the criterion $C_{j}$ for decision maker $P_{k}$. The belief level $b_{i j}^{k}(i=1,2, \ldots, t ; j=1,2, \ldots, m ; k=1,2, \ldots, n)$ belongs to a set of linguistic terms that contain various degrees of preferences required by decision makers $P_{k}$ under the $j^{\text {th }}$ criterion $(j=1,2, \ldots, m)$. The linguistic terms for variable 'preference' are shown in Table 3. Notation '**' can be used to represent that decision maker $P_{k}$ does not know or could not give a belief level for expressing the preference for a solution $S_{i}$ under the criterion $C_{j}$. 
Table 2. Linguistic terms for the comparison of criteria

\begin{tabular}{|l|c|}
\hline Linguistic terms & Fuzzy numbers \\
\hline Absolutely less important & $a_{1}$ \\
\hline Much less important & $a_{2}$ \\
\hline Less important & $a_{3}$ \\
\hline Equally important & $a_{4}$ \\
\hline More important & $a_{5}$ \\
\hline Much more important & $a_{6}$ \\
\hline Absolutely more important & $a_{7}$ \\
\hline
\end{tabular}

Table 3. Linguistic terms for preference belief levels for

\begin{tabular}{|l|c|}
\hline \multicolumn{2}{|c|}{ alternatives } \\
\hline Lowest & $b_{1}$ \\
\hline Very Low & $b_{2}$ \\
\hline Low & $b_{3}$ \\
\hline Medium & $b_{4}$ \\
\hline High & $b_{5}$ \\
\hline Very high & $b_{6}$ \\
\hline Highest & $b_{7}$ \\
\hline
\end{tabular}

Step 6: Elements in each belief level matrix $\left(b_{i j}^{k}\right)$ $(k=1,2, \ldots, n)$ is aggregated into belief vectors $\left(\bar{b}_{j}^{k}\right)$ $(j=1,2, \ldots, m ; k=1,2, \ldots, n)$ by

$$
\bar{b}_{j}^{k}=\widetilde{w}_{j_{1}}^{k} * b_{j j_{1}}^{k}+\widetilde{w}_{j_{2}}^{k} * b_{j j_{2}}^{k}+\cdots+\widetilde{w}_{j_{s}}^{k} * b_{j j_{s}}^{k}
$$

where $b_{j j_{i}}^{k}(i=1,2, \ldots, s)$ is not ' $* *$ '. Based on belief vectors $\left(\bar{b}_{j}^{k}\right)$, decision maker $P_{k}(k=1,2, \ldots, n)$ can have an overall judgment on the alternatives, that is, an individual assessment vector. All individual assessment vectors can compose a group of assessment matrices $\left(\bar{b}_{j}^{k}\right)$.

\section{Stage 3: Group aggregation}

Step 7: Each member $P_{k}(k=1,2, \ldots, n)$ has been assigned with a weight that is described by a linguistic term $\widetilde{v}_{k}, k=1,2, \ldots, n$, as shown in Table 1. A weight vector is obtained: $V=\left\{\widetilde{v}_{k}, k=1,2, \ldots, n\right\}$.

The normalized weight of decision maker $P_{k}(k=$ $1,2, \ldots, n)$ is denoted as:

$$
\widetilde{b}_{k}^{*}=\frac{\widetilde{b}_{k}}{\sum_{i=1}^{n} v_{i 0}^{R}}, \text { for } k=1,2, \ldots, n .
$$

Step 8: Considering the normalized weights of all group members, we can construct a weighted normalized fuzzy decision vector

$$
\left(\widetilde{r}_{1}, \widetilde{r}_{2}, \ldots, \widetilde{r}_{m}\right)=\left(\widetilde{v}_{1}^{*}, \widetilde{v}_{2}^{*}, \ldots, \widetilde{v}_{n}^{*}\right)\left(\begin{array}{cccc}
\bar{b}_{1}^{1} & \bar{b}_{2}^{1} & \ldots & \bar{b}_{m}^{1} \\
\bar{b}_{1}^{2} & \bar{b}_{2}^{2} & \ldots & \bar{b}_{m}^{2} \\
\vdots & \vdots & \ddots & \vdots \\
\bar{b}_{1}^{n} & \bar{b}_{2}^{n} & \cdots & \bar{b}_{m}^{n}
\end{array}\right)
$$

where $\widetilde{r}_{j}=\sum_{k=1}^{n} \widetilde{v}_{k}^{*} \bar{b}_{j}^{k}$.

Step 9: In the weighted normalized fuzzy decision vector the elements $\widetilde{v}_{j}, j=1,2, \ldots, m$, are normalized as positive fuzzy numbers and their ranges belong to the closed interval $[0,1]$. We can then define a fuzzy positive-ideal solution (FPIS, $r^{*}$ ) and a fuzzy negative-ideal solution (FNIS, $r^{-}$) as:

The positive and negative solutions whose distances between each $\widetilde{r}_{j}$ and $r^{*}, \widetilde{r}_{j}$ and $r^{-}$can be calculated as:

$d_{j}^{*}=\mathrm{d}\left(\widetilde{r}_{j}, r^{*}\right)$ and $d_{j}^{-}=\mathrm{d}\left(\widetilde{r}_{j}, r^{-}\right), \quad j=1,2, \ldots, m$,

where $\mathrm{d}(\cdot, \cdot)$ is the distance measurement between two fuzzy numbers. ${ }^{13}$

Step 10: A closeness coefficient is defined to determine the ranking order of all alternatives once the $d_{j}^{*}$ and $d_{j}^{-}$of each $S_{j}(j=1,2, \ldots, m)$ are obtained. The closeness coefficient of each alternative is calculated as:

$$
C C_{j}=\frac{1}{2}\left(d_{j}^{-}+\left(1-d_{j}^{*}\right)\right), \quad j=1,2, \ldots, m .
$$

The alternative $S_{j}$ that corresponds to $\operatorname{Max}\left(C C_{j}, j=\right.$ $1,2, \ldots, m)$ is the most acceptable solution for the decision group.

\subsection{A web-based fuzzy group decision support system for team situation awareness}

A web-based fuzzy GDSS, called WFGDSS, is developed in this study which implements the proposed fuzzy multi-criteria group decision aggregation method to support team situation assessment in a distributed environment. A data management system, a model management system and a user interface are the main components of the system. This 
system allows team members distributed in different locations to participate in situation assessment and decision making activities through the web. It manages the group decision-making process through criteria generation, alternative assessment, opinion interactions and decision aggregation. The working process of using the WFGDSS in team situation assessment is summarized into seven main steps as follows:

Step 1: Set up an online team for assessing a situation.

Step 2: Collect alternatives: members provide their assessment results or awareness for the situation online. Through online discussions and summarizations, a set of them are determined as alternatives.

Step 3: Choose criteria: referring to the criteria received from all members, top-t criteria are determined as the criteria for assessing the situation in the team.

Step 4: Set weights: as team members play different roles and have different experiences, each member is assigned a weight, described by linguistic terms shown in Table 1.

Step 5: Complete criteria comparison matrix: based on the criteria and alternatives determined, each member fills a pairwise comparison matrix of the relative importance of these criteria online using linguistic terms shown in Table 2. The purpose of the step is to obtain weights of all criteria.

Step 6: Complete the belief level matrix: each member fills a belief level matrix online to express the possibility of selecting an assessment result under criteria. That is, to express a member's belief/reference level to an alternative using linguistic terms shown in Table 3.

Step 7: Generate the final assessment result of the team: once all members submit their assessment results and preferences online, the server application software first corrects the inconsistence of each pairwise comparison matrix of the criteria based on linguistic inference rules, then calculates the belief level matrices, the belief vectors, the normalized weights of team members, the weighted normalized fuzzy decision vectors, and the closeness coefficients of all alternatives consecutively. The alternative that corresponds to the maximum closeness coefficient is the aggregated assessment result across the team.

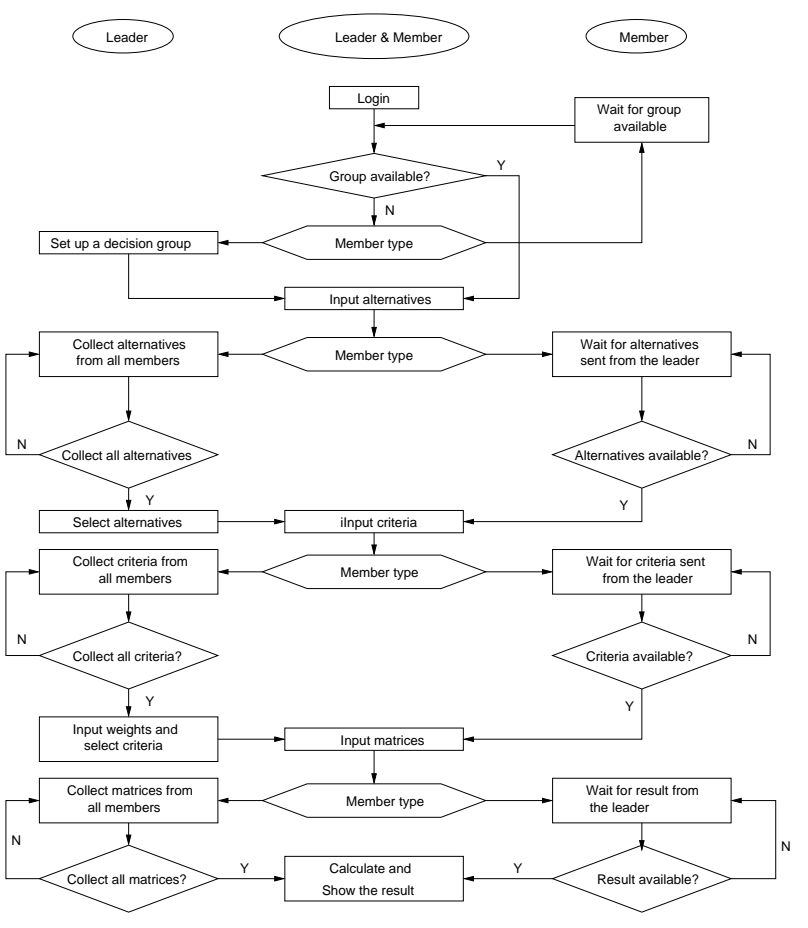

Fig. 1. Flow chart for the WFGDSS working process

Figure 1 shows the working process of team situation assessment using the WFGDSS. Regarding to the process, each member should be aware that understanding of other members' opinions and having interactions with other members have critical to make the process with the system smooth and successful. It involves which stage other members currently are and what they are doing currently, etc. For example, in Step 1, other members will not be able to login to the system until the team leader has set up an assessment team; in Step 7, the process of calculating the final result will not proceed until all members' criteria comparison matrices and belief level matrices are collected.

\section{A Case Study for Team Situation Awareness Supported by the WFGDSS}

In public health risk management, health experts will monitor, predict and respond to emerging epi- 
demiological such as SARS, bird flu to control outbreaks and prevent them from spreading. Suitable early warning systems will then be used to issue alerts when situation assessment results indicate a disease begins to spread. In such a situation, sharing information and SA across a team of health experts will lead to the development of improved coordinated response and decision making to emergencies worldwide. The WFGDSS can become a communication network that allows collaboration among the health experts and observatory around the world working to assess and discover any risk situations.

Suppose a distributed health expert team is collaboratively observing the SARS outbreak and epidemic for a region. The team consists of five members: Officer 1, Officer 2, Officer 3, Officer 4, and Officer 5 who come from different organizations and play different roles in the team. Officer 1 is the Chief Observer to take intellectual responsibility for the surveillance and report. Through collecting and analyzing data from multiple sources, each member is aware of and judges the current situation of the region's SARS epidemic. Each individual's awareness for the situation will share with other members. During the information sharing and integration process, each member can express their own understanding to the current situation and different opinions about which risk level of SARS epidemic is in that region. Their assessments will be integrated into team SA for further activity recommendations such as a suitable SARS alert.

As the data collected and awareness expressed by these team members are by linguistic terms and not consistent, it is hard to directly use the obtained data to determine the risk level of SARS epidemic for the region. The team defines four risk levels: Level 1 (low risk epidemic), Level 2 (middle risk epidemic), Level 3 (high risk epidemic), and Level 4 (very high risk epidemic). These team members need to have consensus awareness on the level of current SARS epidemic in the region through online communication. The developed WFGDSS can support, in some degree, reaching a consensus SA for the team on the risk level of SARS epidemic, which is described as follows.

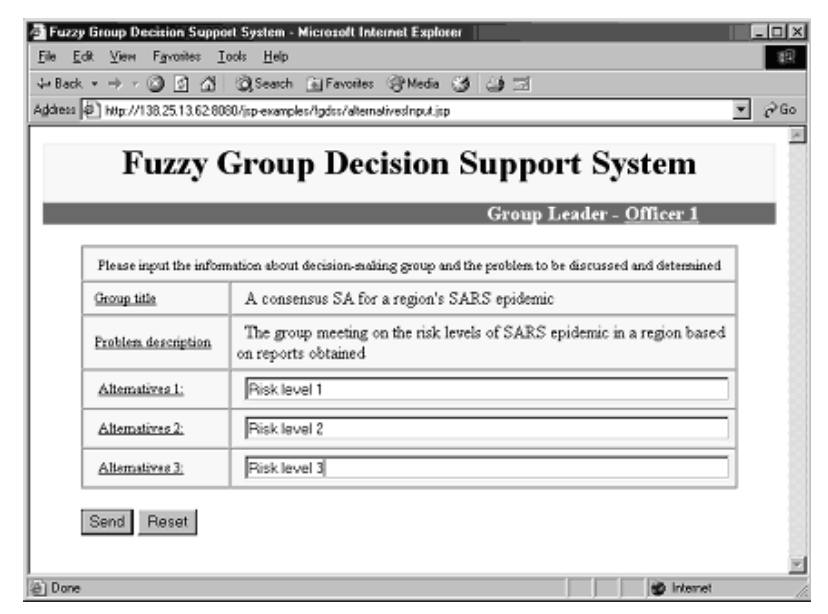

Fig. 2. Three SA alternatives proposed by team members

Step 1: The team leader Officer 1 logins to the system first and sets up an assessment team. A collaboration and information sharing environment is formed.

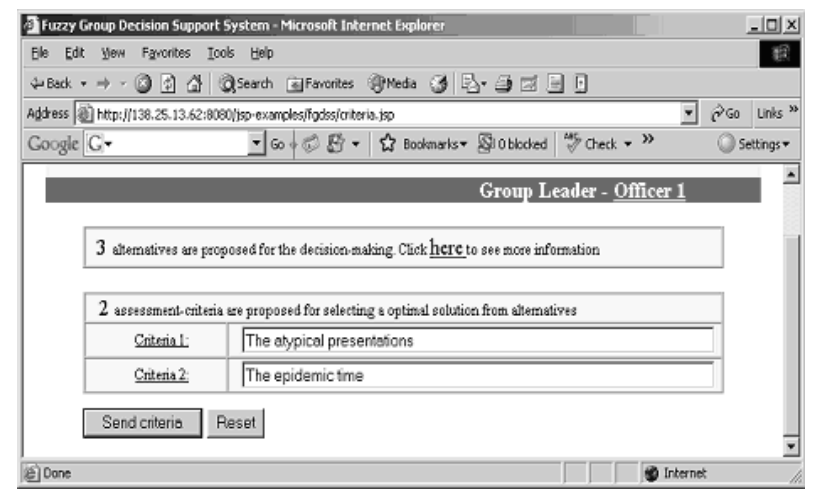

Fig. 3. The criteria proposed by Officer 1

Step 2: All members express their SA about that region's SARS epidemic. Both Officer 1 and Officer 2's believe that the current situation of SARS epidemic in the region is with Risk level 1, Officer 3 and Officer 4's are Risk level 2, and the Officer 5's is Risk level 3. No opinion is for Risk level 4. To support for further discussion and get a consensus SA, the three kinds of opinions are as alternatives for the team SA problem as shown in Fig. 2.

Step 3: Each team member proposes some criteria for assessing these SA alternatives. Officer 1 proposes two criteria that are 'The atypical presentations' and 'The epidemic time' as shown in Fig. 3; 
other officers propose criteria including 'The clinical symptoms and signs', 'The number of infected patients', 'The number of death', and 'The epidemic time'. All these criteria are put into a criterion pool and finally four of them are chosen as criteria, which are as shown in Fig. 4. The information sharing here is very important. It fully uses all members' knowledge and experiences to show their opinions and communicate with each other.

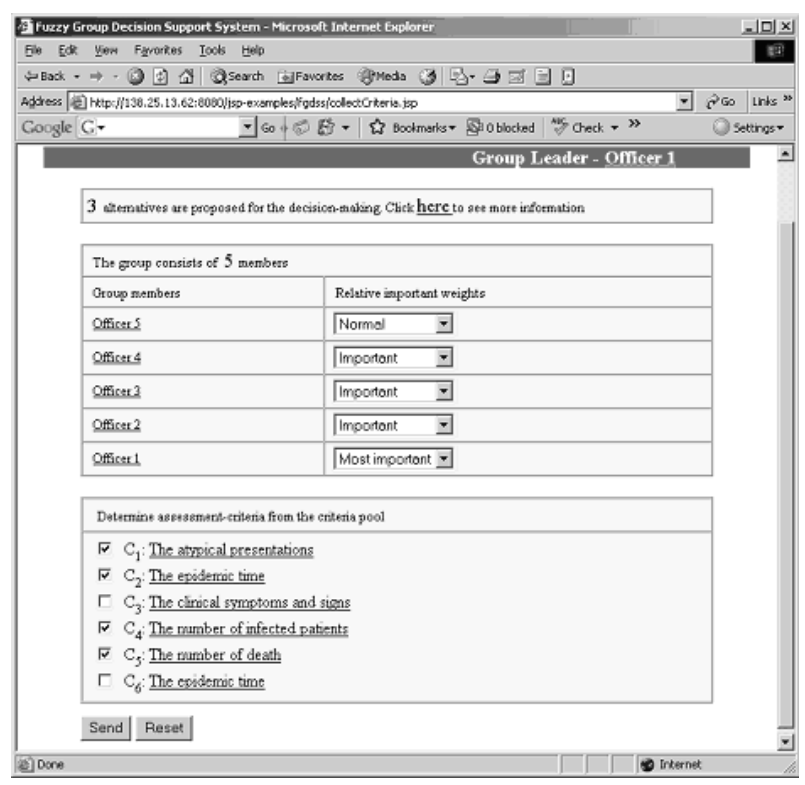

Fig. 4. Four criteria and team members' weights

Step 4: Each member is assigned with a weight that is described by a linguistic term. Here, Officer 1 is assigned as 'Most important', Officer 2, Officer 3, and Officer 4 are assigned as 'Important', respectively, and Officer 5 as 'Normal', which are shown in Fig. 4.

Steps 5-6: Based on the criteria proposed, each team member fills a pairwise comparison matrix of the relative importance of these criteria and a belief level matrix to express their opinion about the current SA under the four selected criteria. Suppose Officer 1 fills the two matrices as in Fig. 5. In the criteria comparison matrix, the criterion 'The atypical presentations' is thought as 'more important' than the criterion 'The epidemic time'; also the criterion 'The number of infected patients' is 'less important' than the criterion 'The number of death', etc. Also in the preference belief level matrix, com- paring with other alternatives under the criteria 'The atypical presentations', the preference belief level of Risk level 1 for the current SA epidemic is regarded as 'very high', Risk level 2 as 'high', Risk level 3 as 'medium', etc. Obviously, team members' preferences are fully expressed here.

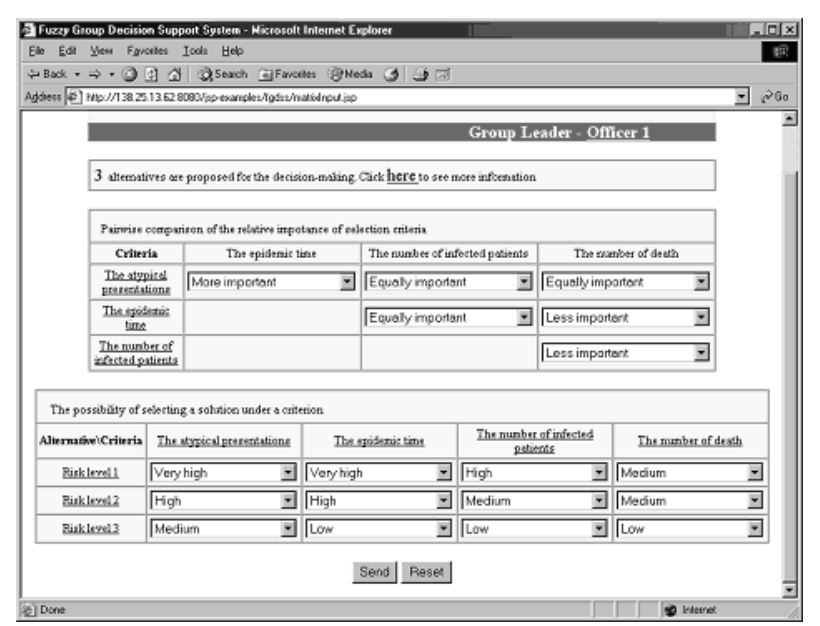

Fig. 5. Criteria comparison matrix and belief level matrix filled by Officer 1

Step 7: Based on the normalized weights of all team members proposed in Step 4, and the criteria comparison matrices and the belief level matrices generated by all members in Steps 5-6, all opinions of the members are aggregated by the method presented in Section 3.2. The final ranking result is as shown in Fig. 6. Based on the result generated, the consensus SA in the team is that the current situation of SARS epidemic in that region is about on Risk level 2.

This WFGDSS also can be used in other situation assessment for risk management and crisis response systems, such as nuclear risk crisis management, pollution response systems and financial early warning systems.

\section{Conclusions and Further Study}

Situation assessment may be the most important and the most difficult stage in crisis decision making process. Part of the difficulty is that measurement of the quality in which situation assessment is concerned. Uncertainty in the measurement is very common in 
various situations such as financial decisions, natural disasters warning decisions, and public health or environmental decisions. In addition, when the concept of SA is extended to teams, the team SA will have the meaning of understanding of the activities of the others, which may affect the whole team's goals and/or procedures. More kinds of uncertainties are involved in situation information and its processing. Particularly, when group members collaborate across distances, they have not only different knowledge and abilities, but also different physical environments. This paper dealt with all these issues in team SA by applying the WFGDSS.

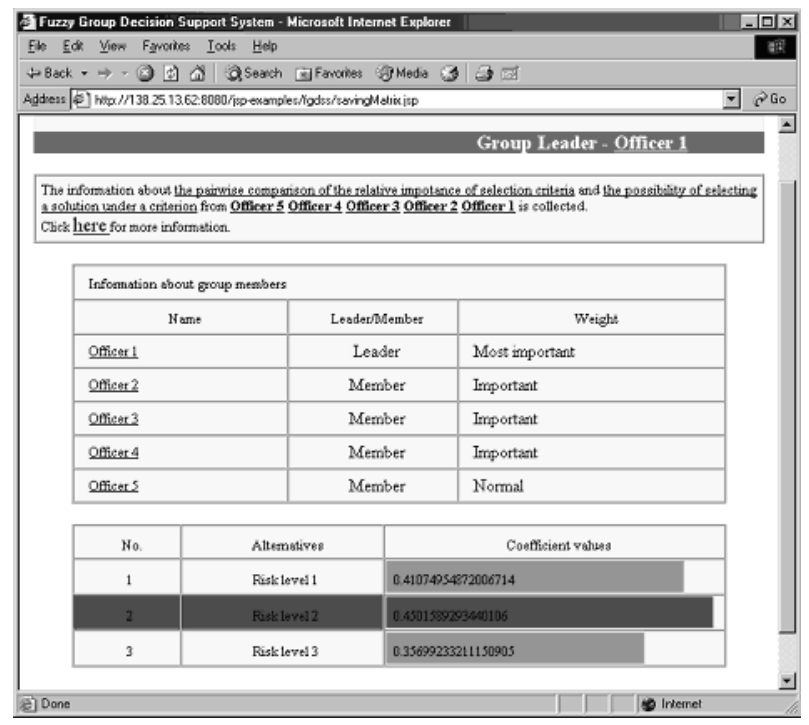

Fig. 6. The final result for the consensus SA in the team

Further study includes the development of approaches of team situation assessment to directly support early warning generation. As team SA forming process is a consequence of the information filtering and coordination, this study will apply target recognition approach 23 to develop suitable systems of information filtering to support early warning generation.

\section{Acknowledgements}

This research was supported in part by the Australian Research Council (ARC) under discovery grants DP0559213 and DP0880739. Authors also thank Ya Gao and Chao Wang for their technical help in completing this paper.

\section{References}

1. M.R. Endsley, "Toward a theory of situation awareness in dynamic systems", Human Factors, 37 32-64 (1995a).

2. E. Salas, C. Prince, D.P. Baker, and L. Shrestha, "Situation awareness in team performance: implications for measurements and training", Human Factors, 37 123-136 (1995).

3. R. Stewart, A.N. Stanton, D. Harris, C. Baber, P. Salmon, M. Mock, K. Tatlock, L. Wells, and A. Kay, Distributed situation awareness in an Airborne Warning and Control System: application of novel ergonomics methodology, Cognition, Technology \& Work, in press, Springer London online, 2007.

4. Y. You and S. Pekkola, "Meeting others - supporting situation awareness on the WWW", Decision Support Systems, 32 71-82 (2001).

5. W. Zhang and R. Hill, "A template-based and patterndriven approach to situation awareness and assessment in virtual humans", Proceedings of the Fourth International Conference on Autonomous Agents, Spain, 116-123 (2000).

6. D.H. Sonnenwald, K.L. Maglaughlin, and M.C. Whitton, "Designing to support situation awareness across distances: an example from a scientific collaboratory", Information Processing \& Management, 40 (2004) 989-1011.

7. M.J. Adams, Y.J. Tenney, R.W. Pew, "Situation Awareness and the Cognitive Management of Complex Systems", Human Factors, 37 85-104 (1995).

8. M.R. Endsley, "Measurement of Situation Awareness in Dynamic Systems", Human Factors, 37 65-84 (1995b).

9. M.R. Endsley, "Theoretical underpinnings of situation awareness: A critical review", Situation Awareness Analysis And Measurement, Mahwah, Lawrence Erlbaum Associates, New Jersey, 2000.

10. M.R. Endsley, B. Bolte, and D.G. Jones, Designing for situation awareness: An approach to user-centered design, Taylor \& Francis, London, 2003.

11. J. Hollan, E. Hutchins and D. Kirsh, "Distributed Cognition: Toward a new foundation for human-computer interaction", ACM Transactions on Computer-Human Interaction, 7 (2) 174-196 (2000).

12. P. Gray, "Group decision support systems", Decision Support Systems, 3 233-242 (1987).

13. J. Lu, G. Zhang, D. Ruan, and F. Wu, Multi-Objective Group Decision Making: methods, software and applications with fuzzy techniques, Imperial College Press, London, 2007.

14. S. Nishida and T. Koiso, "Design of communication 
support system in emergency, Systems, Man, and Cybernetics", 1999, IEEE SMC '99 Conference Proceedings. 1999 IEEE International Conference on, 1, 786791 (1999).

15. L.A. Zadeh, "Fuzzy sets", Information and Control, 8 338-353 (1965).

16. L.A. Zadeh, "The concept of a linguistic variable and its applications to approximate reasoning. Part I, II", Information Sciences 8 199-249, 301-357 (1975), "Part III", Information Sciences 9 43-80 (1975).

17. S. Saint and J.R. Lawson, Rules for Reaching Consensus. A Modern Approach to Decision Makin, JosseyBass, 1994.

18. E. Herrera-Viedma, F. Herrera, and F. Chiclana, "A consensus model for multiperson decision making with different preference structures", IEEE Transactions on Systems, Man and Cybernetics-Part A: Systems and Humans, 32 (3) 394-402 (2002).

19. W. Cook and L. Seiford, "Priory ranking and consen- sus formation", Management Science, 24 1721-1732 (1978).

20. G. Zhang and J. Lu, "An integrated group decision making method with fuzzy preference for alternatives and individual judgments for selection criteria", Group Decision and Negotiation, 12 501-515 (2003).

21. G. Zhang and J. Lu, "Chapter 3: Using general fuzzy number to handle uncertainty and imprecision in group decision-making", Intelligent Sensory Evaluation: Methodologies and Applications, Ruan and Zeng (Eds.), Springer, 51-70 (2004).

22. T.L. Saaty, The Analytic Hierarchy Process, McGrawHill Company, New York, 1980.

23. M.M. Kokar and J. Wang, "An example of using ontologies and symbolic information in automatic target recognition", SPIE Conference on Sensor Fusion: Architectures, Algorithms, and Applications VI, 40-50 (2002). 